University of Wollongong

Research Online

Faculty of Engineering and Information

Faculty of Engineering and Information

Sciences - Papers: Part A

Sciences

$1-1-1999$

\title{
Network reconfiguration for load balancing in distribution networks
}

Kashem M. Muttaqi

Multimedia University, kashem@uow.edu.au

Velappa Ganapathy

Multimedia University

G G. Jasmon

Multimedia University

Follow this and additional works at: https://ro.uow.edu.au/eispapers

Part of the Engineering Commons, and the Science and Technology Studies Commons

Research Online is the open access institutional repository for the University of Wollongong. For further information contact the UOW Library: research-pubs@uow.edu.au 


\title{
Network reconfiguration for load balancing in distribution networks
}

\begin{abstract}
Network reconfiguration of a power distribution system is an operation to alter the topological structure of distribution feeders by changing opedclosed status of sectionalising and tie switches. By transferring loads from the heavily loaded feeders to the lightly loaded ones, network reconfiguration can balance feeder loads and alleviate overload conditions of a network. The branch load-balancing index and the overall system load-balancing index are used to determine the loading conditions of the system and maximum system loading capacity. The index value has to be minimum in the optimal configuration of load balancing. For optimal load balancing condition the branch load-balancing indices in the network are to be more or less equal, and also approximately equal to the system load-balancing index. A general formulation of the network reconfiguration for load balancing is given for the optimal balancing of loads in distribution network and a solution approach is presented. The solution employs a search over different radial configurations, created by considering branch-exchange type switches. The proposed algorithm, called distance measurement technique (DMT) has been developed based on the two-stage solution methodology. The first stage finds a loop, whch gives the maximum improvement in load balancing in the network. In the second stage, a switching option is determined in that loop to obtain maximum improvement in load balancing. The DMT employs a graphical method in which dlfferent circles are drawn and the distances of various points from the centre of the loop circle are computed to achieve the optimal or near optimal configuration for load balancing. The solution algorithm of the proposed method can identify the most effective branch-exchange operations for load balancing with minimum computational effort. The algorithm has been tested with promising results on a 69-bus radial distribution system.
\end{abstract}

\section{Keywords}

distribution, reconfiguration, load, network, balancing, networks

Disciplines

Engineering | Science and Technology Studies

\section{Publication Details}

M. A. Kashem, V. Ganapathy \& G. G. Jasmon, "Network reconfiguration for load balancing in distribution networks," IEE Proceedings - Generation, Transmission and Distribution, vol. 146, (6) pp. 563-567, 1999. 


\title{
Network reconfiguration for load balancing in distribution networks
}

\author{
M.A.Kashem, V.Ganapathy and G.B.Jasmon
}

\begin{abstract}
Network reconfiguration of a power distribution system is an operation to alter the topological structure of distribution feeders by changing open/closed status of sectionalising and tie switches. By transferring loads from the heavily loaded feeders to the lightly loaded ones, network reconfiguration can balance feeder loads and alleviate overload conditions of a network. The branch load-balancing index and the overall system load-balancing index are used to determine the loading conditions of the system and maximum system loading capacity. The index value has to be minimum in the optimal configuration of load balancing. For optimal load balancing condition the branch load-balancing indices in the network are to be more or less equal, and also approximately equal to the system load-balancing index. A general formulation of the network reconfiguration for load balancing is given for the optimal balancing of loads in distribution network and a solution approach is presented. The solution employs a search over different radial configurations, created by considering branch-exchange type switches. The proposed algorithm, called distance measurement technique (DMT) has been developed based on the two-stage solution methodology. The first stage finds a loop, which gives the maximum improvement in load balancing in the network. In the second stage, a switching option is determined in that loop to obtain maximum improvement in load balancing. The DMT employs a graphical method in which different circles are drawn and the distances of various points from the centre of the loop circle are computed to achieve the optimal or near optimal configuration for load balancing. The solution algorithm of the proposed method can identify the most effective branch-exchange operations for load balancing with minimum computational effort. The algorithm has been tested with promising results on a 69-bus radial distribution system.
\end{abstract}

\section{Introduction}

Distribution systems are normally configured radially for effective co-ordination of their protective systems. Networks are reconfigured to reduce the system power loss (network reconfiguration for loss reduction), and to relieve overloads in the networks (network reconfiguration for load balancing). This operation transfers loads from one feeder to another, which will significantly improve the operating condition of the overall system. It is required to reconfigure the network from time to time, since distribution lines show different characteristics as each of the distribution feeders consists of residential, commercial, industrial, etc. type of load. In addition, some parts of the distribution system become heavily loaded at certain time of the day and lightly loaded at other times. For load balancing the loads are required to be rescheduled more efficiently by modifying the radial structure of the distribution feeders.

Aoki et al. $[1,2]$ formulated the load balancing and service restoration problems by considering the capacity and voltage constraints as a mixed integer nonlinear optimisa-

\section{(C) IEE, 1999}

IEE Proceedings online no. 19990694

DOI: 10.1049/ip-gtd:19990694

Paper first received 18th February and in revised form 19th July 1999

The authors are with the Multimedia University, 63100 Cyberjaya, Selangor $\mathrm{D} / \mathrm{E}$, Malaysia tion problem and converted the problem into a series of continuous quadratic programming subproblems. Baran and $\mathrm{Wu}$ [3] formulated the problem of loss minimisation and load balancing as an integer programming problem. H.D. Chiang et al. [4] proposed a constrained multiobjective and nondifferentiable optimisation problem with equality and inequality constraints for both loss reduction and load balancing. Y.H. Song et al. [5] used fuzzy controlled evolutionary programming to reconfigure the distribution network for loss reduction. G. Peponis et al. [6] developed an improved switch-exchange method for load balancing problem, using switch exchange operations. M.A. Kashem et al. [7] proposed a new load-balancing index and applied it to the network for load balancing. Whei-Min Lin et al. [8] presented a new load balancing algorithm for the threephase unbalanced distribution systems.

In this paper, a network reconfiguration based branchexchange algorithm for load balancing is proposed. Initially, a graphical representation is used to determine the loop that gives the maximum improvement in load balancing. Then the switching operation to be executed in that loop to get the maximum improvement in load balancing in the network is identified. Using the proposed search technique, the network configuration that optimally or near optimally improves the load balancing can be achieved with reduced computational effort. The proposed method is applicable in networks with the possibility for on-line changing of the topological network structure. 


\section{Formulation of load balancing problem}

An objective function for load balancing is presented which consists of two components. One is the branch load-balancing index and the other is the system load-balancing index [4]. Branch load-balancing index $L B_{i}$ is defined as a measure of how much a branch can be loaded without exceeding the rated capacity of that branch. This can be represented mathematically as

$$
L B_{i}=\frac{S_{i}}{S_{i}^{\text {max }}}
$$

where $S_{i}$ is the complex power flowing through the branch $i$ and $S_{i}^{\max }$ is the maximum capacity or rating of branch $i$. The system load balancing index $L B_{s y s}$ of the entire system is represented as

$$
L B_{\text {sys }}=\frac{1}{n_{b}} \sum_{i=1}^{n_{b}} \frac{S_{i}}{S_{i}^{\max }}
$$

where $n_{b}$ is the total number of branches in the system. The objective is to optimise the branch load indices so that the system load-balancing index is minimised. In other words, all the branch load-balancing indices $L B_{i}$ are set to be more or less the same value and is also nearly equal to the system load balancing index $L B_{s y s}$. This can be represented mathematically

$$
\frac{S_{1}}{S_{1}^{\text {max }}} \cong \frac{S_{2}}{S_{2}^{\text {max }}} \cong \cdots \cong \frac{S_{n}}{S_{n}^{\text {max }}} \cong \frac{1}{n_{b}} \sum_{i=1}^{n_{b}} \frac{S_{i}}{S_{i}^{\text {max }}}
$$

The objective function is to be realised subject to the following constraints:

- The system loss must be minimised, i.e.

$$
\min \sum_{i=1}^{n_{b}} r_{i} \frac{P_{i}^{2}+Q_{i}^{2}}{\left|V_{i}\right|^{2}}
$$

where $P_{i}$ is the real power flow, $Q_{i}$ is the reactive power flow, $V_{i}$ is the node voltage and $r_{i}$ is the resistance of the line $i$.

- The voltage magnitude of each node must be within a permissible range, i.e. $\left|V_{i}^{\min }\right| \leq\left|V_{i}\right| \leq\left|V_{i}^{\max }\right|$.

The critical condition of each branch is the point at which the load balancing index $L B_{i}$ of that branch is equal to 1 , beyond which the branch rated capacity is exceeded. Though it is not practically possible to make all the branch load-balancing indices $L B_{i}$ exactly equal, it is possible that by using network reconfiguration and adjusting the loadbalancing indices, the load balancing in the overall system can be improved.

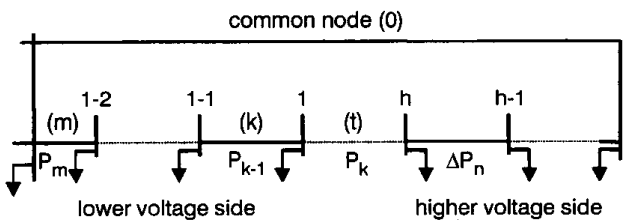

Fig. 1 Loop associated with tie-line $t$

\section{Proposed distance measurement technique}

In a radial distribution network several loops can be formed by closing the tie-switches in the network, and the number of loops is equal to the number of tie-lines. A loop in a radial network is created by a tie-line $t$, as shown in Fig. 1. There is a voltage difference across the normally open tie switch in the tie-line. The higher voltage drop side of the tie switch is called the lower voltage side (lv-side) and the lower voltage drop side of the tie switch is called higher voltage side (hv-side) of the loop. The lower voltage and higher voltage sides in the Figure are denoted by $l$ and $h$, respectively.

Consider the branch exchange between branches $t$ and $m$, where $t$ is the open branch and $m$ is the closed branch. By opening the branch $t$ the loop is divided into two parts, the first part of which consists of the branches in the loop that extends between nodes 0 to 1 , and the second part of the branches extends between nodes 0 to $\mathrm{h}$. The change in load balancing due to this branch exchange is derived in the Appendix (Section 7) and written as

$$
\begin{aligned}
\Delta L B_{t m}^{S}= & 2 P_{m}\left(\sum k_{l} P_{l}-\sum k_{h} P_{h}\right) \\
& +2 Q_{m}\left(\sum k_{l} Q_{l}-\sum k_{h} Q_{h}\right) \\
& -\left(P_{m}^{2}+Q_{m}^{2}\right) k_{\text {loop }}
\end{aligned}
$$

where $\Delta L B_{t m}^{S}$ is the change in load balancing due to branch exchange $t-m$

$P_{m}$ and $Q_{m}$ are real and reactive power flows in branch $m$ (to be removed), respectively

$k_{i}$ is the inverse of the square of the maximum capacity of a branch $i$

$k_{l} P_{l}$ and $k_{h} P_{h}$ are products of $k$ and $P$ on the lv-side $l$ and hv-side $h$ of the loop, respectively

$k_{\text {loop }}$ is the sum of $k$ value of each branch in the loop in which power-flow exists.

If the value of $\Delta L B_{t m}^{S}$ is greater than zero the load balancing is improved, and if it is less than zero the load balancing is deteriorated. The equation for the change in load balancing derived in this paper is similar to the equation for power loss reduction obtained by Baran and $\mathrm{Wu}$ [3]. Hence the accuracy analysis for load balancing can be carried out in the same way as in [3].

\subsection{Determination of loop which gives maximum load balancing}

In the proposed technique distances of various points $\left(P_{m}\right.$, $Q_{m}$ ) from the centre of the loop circle are calculated. The loop circle is drawn from the relationship between the load balancing $\Delta L B_{t m}^{S}$ and the power transfer $\left(P_{m}, Q_{m}\right)$. The polarity of the nominal load balancing in each loop is important for this technique. The nominal branch is the first adjacent branch to the tie branch on the lv-side of the loop. In the loop, as shown in Fig. 1, the nominal branch is $k$. If the nominal load balancing $\Delta L B_{t k}^{S}$ is negative, then there is no branch in the loop that can be a candidate for a branch exchange. If the nominal load balancing $\Delta L B_{t k}^{S}$ is positive, there is a branch that can be a candidate for branch exchange and the branch to be opened should be the one that optimises $\Delta L B_{t m}^{S}$. This fact is verified by analysing all the possible branch exchanges in various

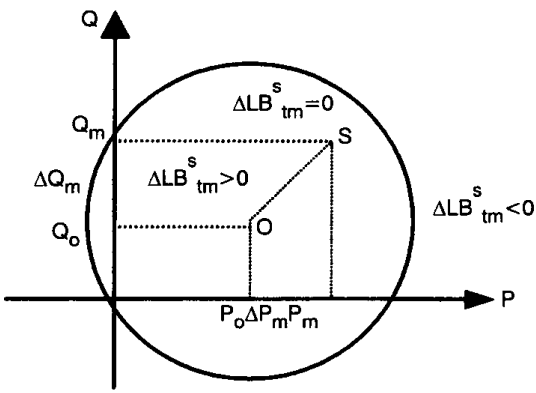

Fig.2 Zero load balancing loop circle

IEE Proc.-Gener. Transm. Distrib., Vol. 146, No. 6, November 1999 
loops. The nominal power flow $\left(P_{k}, Q_{k}\right)$ can be represented as a point on the $\mathrm{P}-\mathrm{Q}$ plane where a loop circle is drawn as shown in Fig. 2. The distance between the centre of the loop circle and the nominal point is given by $\Delta D C_{t k}$ and it is used to identify the nominal load balancing type (+ve, ve or 0 ) as

- If $\Delta D C_{t k}>\left(A^{2}+B^{2}\right)^{1 / 2} / C$ the nominal point is outside the loop circle and hence nominal load balancing $\Delta L B_{t k}^{S}$ is negative,

- If $\Delta D C_{t k}=\left(A^{2}+B^{2}\right)^{1 / 2} / C$ the nominal point is on the loop circle and hence nominal load balancing $\Delta L B_{t k}^{S}$ is zero,

- If $\Delta D C_{t k}<\left(A^{2}+B^{2}\right)^{1 / 2} / C$ the nominal point is inside the loop circle and hence nominal load balancing $\Delta L B_{t k}^{S}$ is positive.

Eqn. 4 can be written in the following form:

$$
\Delta L B_{t m}^{S}=2 A P_{m}+2 B Q_{m}-C\left(P_{m}^{2}+Q_{m}^{2}\right)
$$

where $A=\Sigma k_{l} P_{l}-\Sigma k_{h} P_{h}, B=\Sigma k_{l} Q_{l}-\Sigma k_{h} Q_{h}$, and $C=$ $k_{\text {loop. }} A, B, C$ are constants for a particular loop and are not functions of the variables $P_{m}, Q_{m}$ of that loop. Using eqn. 5 and separating the $P_{m}$ and $Q_{m}$ terms,

$$
\begin{aligned}
\Delta L B_{t m}^{S}= & \left(A^{2}+B^{2}\right) / C \\
& -C\left\{\left(P_{m}-A / C\right)^{2}+\left(Q_{m}-B / C\right)^{2}\right\}
\end{aligned}
$$

Eqn. 6 can be represented by a circle with the centre at $\left(P_{m_{-} \text {loop }}, Q_{m_{-} \text {loop }}\right)$, where $P_{m_{\text {loop }}}$ and $Q_{m_{\text {loop }}}$ are the values which give the maximum load balancing in a loop for which the values of $P_{m_{-} \text {loop }}$ and $Q_{m_{-} \text {loop }}$ are $A / C$ and $B / C$, respectively. Substituting these values into eqn. 5 , the maximum value of $\Delta L B_{l m}^{S}$ in the loop is obtained as

$$
\Delta L B_{\text {tm_loop }}^{S}=\left(A^{2}+B^{2}\right) / C
$$

For zero load balancing the radius of the circle is $\left(A^{2}+\right.$ $\left.B^{2}\right)^{1 / 2} / C$ and if the improvement in load balancing occurs in the loop tne radius will decrease. Rearranging eqn. 6 we get the modified loop circle as

$$
\left[P_{m}^{\prime}-\frac{A}{\sqrt{C}}\right]^{2}+\left[Q_{m}^{\prime}-\frac{B}{\sqrt{C}}\right]^{2}=\frac{A^{2}+B^{2}}{C}
$$

where $P_{m}^{\prime}=(\sqrt{ } C) P_{m}$ and $Q_{m}^{\prime}=(\sqrt{ } C) Q_{m}$. The radius of the above circle is $\left[\left(A^{2}+B^{2}\right) / C\right]^{1 / 2}$, which is the square root of $\Delta L B_{\text {tm loop }}^{S}$ in eqn. 7. The modified loop circles can be drawn using eqn. 8 for all the loops in the system and the largest circle will give the maximum load-balancing loop among all the circles drawn for the loops in the network.

\subsection{Determination of switching-option to be performed for load balancing}

After determining the loop that would give the maximum load balancing, distances of various points $\left(P_{m}, Q_{m}\right)$ from the centre of the loop circle are calculated to find the branch to be exchanged with the tie-line for the maximum improvement. This technique calculates distances from the centre of the loop circle to all the points that correspond to the branches and their respective power flows. The lowest distance is considered the best for maximum load balancing and is chosen as the candidate for improvement by exchanging the corresponding branch with the tie-line. To explain the concept of the distance measurement technique, eqn. 6 is used which is graphically represented by the circle shown in Fig. 2. It is drawn from the relationship between the load balancing $\triangle L B_{t m}^{S}$ and the power transfer $\left(P_{m}, Q_{m}\right)$ by considering the zero load balancing (i.e. $\Delta L B_{t m}^{S}=0$ ).
Therefore the circle is called zero load balancing loop circle or simply loop circle. Assume that the centre of this circle is $O\left(P_{o}, Q_{o}\right)$. The equations for the centre of the zero load balancing loop circle $P_{o}$ and $Q_{o}$ are found as follows:

$$
\begin{gathered}
P_{o}\left(=P_{m_{-} \text {loop }}\right)=\left(\sum k_{l} P_{l}-\sum k_{h} P_{h}\right) / k_{\text {loop }} \\
Q_{o}\left(=Q_{m_{\text {loop }}}\right)=\left(\sum k_{l} Q_{l}-\sum k_{h} Q_{h}\right) / k_{\text {loop }}
\end{gathered}
$$

It has been mentioned that the individual branch power flows $\left(P_{m}, Q_{m}\right)$ in the loop are represented as several points in the $P-Q$ plane. It is proven that load balancing is improved for the points which are inside the loop circle and correspond to the branch power flows in the lv-side of the loop. Therefore let us assume that $S\left(P_{m}, Q_{m}\right)$ is one of such points. The distance from centre of the loop circle $O$ to this point $S$ is given by

$$
\Delta D C_{t m}=\sqrt{\Delta P_{m}^{2}+\Delta Q_{m}^{2}}
$$

where $\Delta D C_{t m}=$ distance from the centre of the loop circle to a point that corresponds to a branch-exchange $\Delta P_{m}=$ $P_{m}-P_{o}$ and $\Delta Q_{m}=Q_{m}-Q_{o}$.

As described, only the point at the centre of the loop circle can maximise the load balancing for that loop. In practical cases, the point may not lie at the centre of the loop circle and only the point nearest to the centre of the loop circle will maximise the load balancing in the loop. In other words, the minimum value of $\Delta D C_{t m}$ represents the maximum value of load balancing. Therefore the point that gives a minimum value of $\triangle D C_{t m}$ corresponds to the branch to be exchanged with the tie-line in the loop and gives maximum load balancing in the loop. To obtain a minimum value of $\Delta D C_{t m}$, all the points that correspond to the branch exchanges in the lv-side of the loop will be checked and the lowest distance will be obtained.

\subsection{Solution procedure}

The procedure to determine which loop gives the maximum load balancing is summarised as

(i) Run the load flow program to obtain the power flow in branches

(ii) Check the nominal load balancing for every loop in the system and select the loops with positive nominal load balancing

(iii) If there is no positive nominal load-balancing loop, stop. Otherwise, go to the next step for determining the maximum load balancing loop

(iv) By using eqn. 8, draw the modified loop circle for each loop obtained from step (ii)

(v) Identify the largest circle and select the corresponding loop which gives the maximum load balancing.

Having found the loop which would give the maximum load balancing, the branch to be exchanged is determined as:

(vi) For the selected loop compute the centre of the loop circle using eqns. 9 and 10.

(vii) Using eqn. 11, calculate the distances $\triangle D C_{t m}$ from the centre $\left(P_{o}, Q_{o}\right)$ to all the branch flows $(P, Q)$ in the lv-side of the loop.

(viii) The switching options that correspond to the values of $\Delta D C_{t m}$ computed in step (vii) are ranked in ascending order.

(ix) Check the switching option from the top of the list for constraint violation: voltage limit and system loss as found from the load-flow solution. If all the constraints are satis- 
fied, go to step (x). Otherwise, select the next ranking switching option for which the constraints are satisfied. If no branch is found, go to step (xi).

(x) Select this as a switching option for branch exchange.

(xi) Repeat steps (i) to (vii) using the selected switching option.

\section{Test results and discussion}

The test system is a hypothetical $12.66 \mathrm{kV}$ radial distribution system with 69 buses and seven laterals, which has been derived from a portion of the PG\&E distribution system [9]. The sizes of conductors of the lines have been designed based on the line parameters given in the network, and the maximum capacity ( $\mathrm{kVA}$ rating) of each line is found. The schematic diagram of the test system is shown in Fig. 3 and the data of the test system used in this paper are the same as in $[7,9]$. There are five tie-lines (looping branches) in the system, and a sectionalising switch on every branch of the system. The total system loads for the base configuration are $3802.19 \mathrm{~kW}$ and $2694.60 \mathrm{kVAr}$.

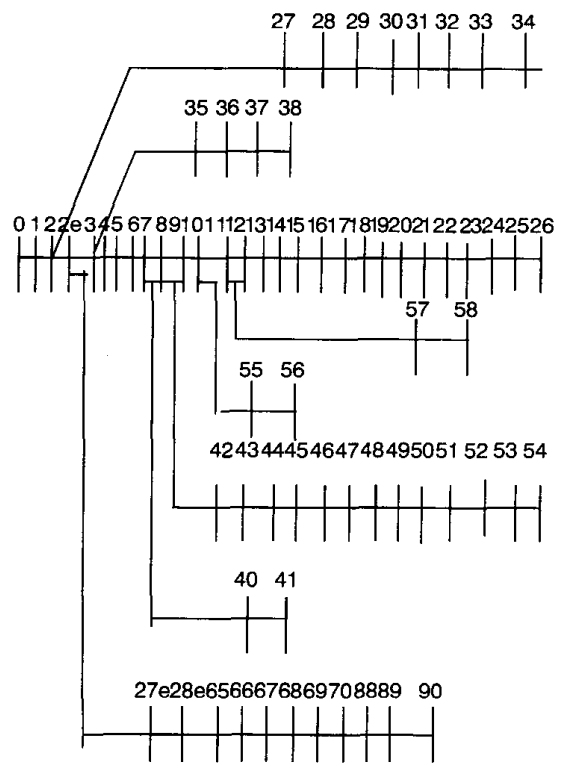

Fig.3 Schematic diagram of 69-bus distribution system

Table 1: Test results

\begin{tabular}{|c|c|c|c|}
\hline \multirow[b]{2}{*}{ Search level } & \multicolumn{3}{|l|}{ Proposed DMT } \\
\hline & $\begin{array}{l}\text { Maximum load } \\
\text { balancing loop* }\end{array}$ & $\begin{array}{l}\text { Branch } \\
\text { exchange } \\
\text { (in-out) }\end{array}$ & $\begin{array}{l}\text { System index } \\
\text { value } L B_{\text {sys }}\end{array}$ \\
\hline Base config. & & & 0.03456 \\
\hline 1 & loop 4 & $73-59$ & 0.02960 \\
\hline 2 & $\operatorname{loop} 3$ & $72-13$ & 0.02879 \\
\hline 3 & loop 5 & $74-62$ & 0.02782 \\
\hline 4 & loop 3 & $13-15$ & 0.02781 \\
\hline \multirow[t]{3}{*}{5} & no loop is selected ${ }^{* *}$ & & \\
\hline & selection of final branc & -exchange & \\
\hline & $73-59,72-15,74-62$ & & \\
\hline
\end{tabular}

Note: '(in-out)' indicates branch into the system and branch out from the system respectively.

* loop 1, loop2, loop3, loop4 and loop5 are associated with tie-lines $70,71,72,73$ and 74 , respectively.

** No loop is selected in the search due to the negative nominal load balancing in each loop.

566
The test results are tabulated in Table 1 . In the proposed algorithm the optimal network configuration for load balancing is achieved after five search levels. At each search level (or iteration) a load flow solution is obtained, the maximum load-balancing loop is selected and a branch exchange is determined. As shown in Table 1 each of the minimal tree search solutions at the first iteration of the proposed algorithm has found loop 4 as the maximum load balancing loop, and the branch exchange 73-59 to be performed in that loop to achieve maximum improvement in load balancing. Fig. 4 shows a graphical determination by which loop 4 is found to give the maximum improvement in load balancing in the proposed distance measurement technique. The test results of Table 1 also indicate that the first branch exchange contributes most of the improvement in load balancing. Degrees of load balancing at different search levels are shown in Table 1, in which $73.48 \%$ of the total improvement in load balancing is obtained by the first branch exchange (or at first search level). The CPU time for the proposed method has been found to be 6.43 seconds using Pentium II (350MHz) processor. In the method of [3] a load-flow solution is required for every branch exchange, whereas the proposed method has drastically reduced the number of load-flow runs as well as CPU time because of the determination of the maximum load balancing loop and then the branch exchange in that loop. The features of the proposed method are also compared with that of [8] and summarised in Table 2.

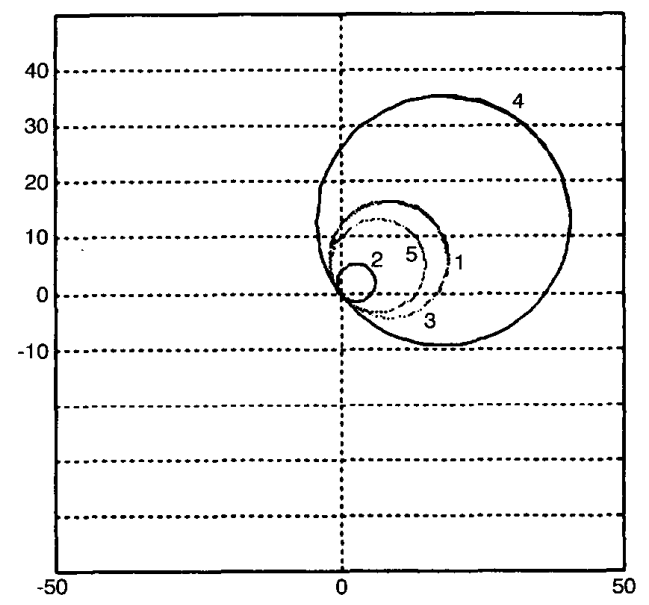

Fig.4 Modified loop circles are drawn

Table 2: Comparative study

\begin{tabular}{lll}
\hline Proposed method & Method in [8] \\
\hline 1 & $\begin{array}{l}\text { Constraints such as voltage } \\
\text { limit and system loss are } \\
\text { considered for finding optimal } \\
\text { solution }\end{array}$ & $\begin{array}{l}\text { Constraints are not imposed } \\
\text { explicitly and hence the } \\
\text { solution may lead to increased } \\
\text { system loss and/or violating } \\
\text { voltage limit. }\end{array}$ \\
2 & $\begin{array}{l}\text { Easily applied to any large } \\
\text { system in practice as it is able } \\
\text { to determine optimal solution } \\
\text { with minimum computational } \\
\text { effort and time, proven for } \\
\text { 69-bus test system. }\end{array}$ & $\begin{array}{l}\text { Search will be exhaustive, } \\
\text { especially for larger system } \\
\text { effort and time will be } \\
\text { much higher. Test system } \\
\text { considered is a small } \\
\text { hypothetical system. }\end{array}$ \\
$\begin{array}{l}\text { Load balancing of every } \\
\text { branch in each feeder has } \\
\text { been considered. }\end{array}$ & $\begin{array}{l}\text { Load balancing of every } \\
\text { feeder only is considered. }\end{array}$ \\
\hline
\end{tabular}

IEE Proc.-Gener. Transm. Distrib., Vol. 146, No. 6, November 1999 
Fig. 5 shows the voltage profile giving the improvement achieved by the proposed technique for load balancing. As shown, most of the bus voltages have been improved after network reconfiguration for load balancing. The test results show that before reconfiguration, the minimum bus voltage was 0.9092 p.u. at the end of line 65 , and after reconfiguration it has increased to $0.9428 \mathrm{p}$.u. at the end of line 61 .

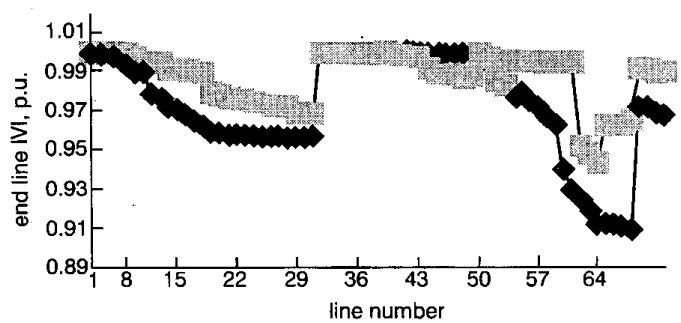

Fig. 5 Voltage profile

$$
\text { - initial }
$$

\section{Conclusions}

The problem of network reconfiguration in the context of network load balancing has been addressed and a new solution approach proposed. A graphical method is used in which several circles are drawn representing the changes in the load balancing. The loop that gives the maximum load balancing is identified as the largest circle amongst the modified loop circles drawn for all the loops. The loop circle is drawn for the loop identified and the distances of the various points $\left(P_{m}, Q_{m}\right)$ from the centre of the loop circle are measured. Then the lowest distance is found and the corresponding branch is used for branch-exchange to achieve maximum load balancing in the network. The developed technique eliminates the need to consider the switching exchange in other loops of the system, and also reduce the numerous load flow studies, thereby significantly reducing the computational requirements. To reflect the efficiency and performance of the proposed method for reducing the computational complexity and the large dimensionality of the problems, a large system with 69-bus and seven laterals has been used as the test system. Test results indicate that the method can identify the most effective branch-exchanges for improvement in load balancing. It is found that the optimal or near optimal configuration for load balancing also improves the voltage profile of the network.

\section{References}

1 AOKI, K., KUWABARA, H., SATOH, T., and KANEZASHI, M.: 'An efficient algorithm for load balancing of transformers and feeders', IEEE Trans., 1988, PWRD-3, (4), pp. 1865-1872

2 AOKI, K., ICHIMORI, T., and KANEZASHI, M.: 'Normal state optimal load allocation in distribution systems', IEEE Trans. Power Deliv., 1987, 3, (1), pp. 147-155

3 BARAN, M.E., and WU, F.F.: 'Network reconfiguration in distribution systems for loss reduction and load balancing', IEEE Trans., 1989, PWRD 4, (2), pp. 1401-1407

4 CHIANG, H.D., and JUMEAU, R.J.: 'Optimal network reconfigurations in distribution systems: Part 1: A new formulation and a solution
methodology', IEEE Trans., 1990, PWRD-5, (4), pp. 1902-1909

5 SONG, Y.H., WANG, G.S., JOHNS, A.T., and WANG, P.Y.: 'Distribution network reconfiguration for loss reduction using fuzzy controlled evolutionary programming', IEE Proc., Gener., Transm. Distrib., 1997, 144, (4), pp. 345-350

6 PEPONIS, G., and PAPADOPOULOS, M.: 'Application of heuristic methods on large-scale networks', IEE Proc., Gener., Transm. Distrib., 1995, 142, (6), pp. 631-638

7 KASHEM, M.A., and MOGHAVVEMI, M.: 'Maximising radial voltages stability and load balancing via loss minimisation in distribution networks'. Proceedings of the international conference on Energy management and power delivery (EMPD'98), Singapore, 3-5 March 1998, pp. $91-96$

8 LIN, W.-M., and CHIN, H.-C.: 'A current index based load balancing technique for distribution systems'. Proceedings of the international conference on Power system technology, (POWERCON'98), Aug. 1998, pp. 223-227

9 BARAN, M.E., and WU, F.F.: 'Optimal capacitor placement on radial distribution systems', IEEE Trans., 1989, PWRD-4, (1), pp. $725-734$

\section{Appendix}

\subsection{Derivation of 'change in load balancing' formula}

The squared value of the load-balancing index $L B_{i}^{S}$ can be defined as

$$
L B_{i}^{S}=\frac{S_{i}^{2}}{\left(S_{i}^{\text {max }}\right)^{2}}=\frac{P_{i}^{2}+Q_{i}^{2}}{\left(S_{i}^{\text {max }}\right)^{2}}=k_{i}\left(P_{i}^{2}+Q_{i}^{2}\right)
$$

Then for the whole system the total load balancing, $L B_{n e t} S$ can be found as

$$
L B_{n e t}^{S}=\sum_{i=1}^{n_{b}} L B_{i}^{S}=\sum_{i=1}^{n_{b}} k_{i}\left(P_{i}^{2}+Q_{i}^{2}\right)
$$

Eqn. 13 is analogous to eqn. 8 of [3]. Therefore the derivation of the change in load balancing is analogous to the derivation of the change in loss reduction of [3]. The change in load balancing for the branch-exchange $t-m$ can be obtained as

$$
\begin{aligned}
\Delta L B_{t m}^{S}= & 2 P_{m}\left(\sum k_{l} P_{l}-\sum k_{h} P_{h}\right) \\
& +2 Q_{m}\left(\sum k_{l} Q_{l}-\sum k_{h} Q_{h}\right) \\
& -\left(P_{m}^{2}+Q_{m}^{2}\right) k_{\text {loop }}
\end{aligned}
$$

